\title{
The effects of asymmetric liking on the attribution of dominance in dyads
}

\author{
EILEEN G. THOMPSON and JAMES L. PHILLIPS \\ Michigan State University, East Lansing, Michigan 48824
}

\begin{abstract}
This study explored a feature of what might be called "implicit theories of interpersonal relations." The question addressed concerns the nature of the interrelation that subjects placed on the violation of a salient schema which they held for dyadic affective relations. When presented with information concerning the degree of asymmetry of liking in a dyad, subjects inferred greater dominance on the part of the better liked person. This result is consistent with the view that subjects attend to structural characteristics of interpersonal relationships as cues in the interpretation of those relations.
\end{abstract}

An important topic in psychological research has centered upon the conception of an "implicit theory of personality." By and large, what has been meant by that phrase is the set of expectations which the person has about the co-occurrence of traits or other characteristics in others or, more generally, about the structural organization of personal descriptors which the person assumes in interpersonal relationships (e.g., Rosenberg $\&$ Sedlak, 1972).

Paralleling this work have been investigations exploring what might be called "implicit theories of interpersonal relations." An implicit theory of interpersonal relations defines the set of assumptions or expectations which the person has about interpersonal interaction.

One area of interpersonal expectations has been explicated by balance theory (Cartwright \& Harary, 1956; Heider, 1958). Applicable to relationships involving affect or liking, balance theory involves the expectations that such relations are symmetric (if Joe likes Bill, then Bill will like Joe), that relations involving positive affect are transitive (if Sue likes Jane and Jane likes Nancy, then Sue will like Nancy), and that relations involving negative affect are intransitive (if Joe dislikes Bill and Bill dislikes Fred, then Joe will like Fred). The conjunction of these three assumptions might be characterized as a cognitive schema summarizing interpersonal expectations about affective relations.

Interpersonal relations involving dominance or control are thought to be subject to a different sort of schema. DeSoto and Kuethe (1959) have characterized such expectations as conforming to a linear ordering schema. This schema is the conjunction of the assumptions that relations are asymmetric (if Sue dominates Jan, then Jan submits to Sue) and transitive (if Bill dominates Joe and Joe dominates Fred, then Bill dominates Fred).

Previous work has shown that these two schemata tend to be appropriate for affective and control relations, respectively. This has been the case when a person is asked to predict the nature of an interpersonal relationship (e.g., Blumberg, 1969; Burnstein, 1967; DeSoto
\& Kuethe, 1959) and when the person is asked to learn a social structure (e.g., DeSoto, 1960; Press, Crockett, \& Rosenkrantz, 1969; Walker, 1976; Zajonc \& Burnstein, 1965).

One area of investigation which has not received attention has been the interaction of affect and dominance. That is, if information is available concerning interpersonal affect, how does the person draw inferences concerning interpersonal dominance? In particular, if affective relations-in a particular instance-fail to conform to the appropriate schema, then does this lead to inferences relating to control?

An important way in which schemata for affect and for dominance differ is with respect to symmetry. The balance schema demands that affective relations be symmetric, while the linear ordering schema posits that dominance relations be asymmetric. If these schematic properties are indeed important characteristics in the person's recognition of and response to types of interpersonal relations, then it is reasonable to expect that counterschematic information will give rise to crossrelational inferences. That is, if one knows that Person A dominates Person B and that Person B dominates Person $A$, this counterschematic information may lead to the inference that affect is somehow involved in the relationship between A and B. Similarly, knowing that Person A likes Person B but that Person B dislikes Person A may lead to inferences concerning interpersonal influence.

The present paper reports on a preliminary investigation of inferences which result from the presentation of counterschematic information. In particular, we have examined the propensity to infer dominance or control in affective relationships as a function of the degree of asymmetry.

\section{METHOD}

\section{Subjects}

Subjects in this study were 20 male and 20 female undergraduates enrolled in introductory psychology classes at Michigan State University. The subjects participated in exchange for 
course credit. Subjects came to the laboratory in small groups but worked on the task individually.

\section{Materials and Procedure}

Each subject was introduced to the task through an oral explanation and then was given the test booklet, the first page of which contained instructions. The instructions stated that the subject was to consider a number of different liking relationships between two imaginary people and was then to answer questions about the probable nature of these relationships.

At the top of each page of the booklet, two sentences were given. The first sentence described the degree of liking from the first imaginary person to the second; the second sentence described the degree of liking from the second person to the first. Two types of affect were used (liking, disliking), and the relation was modified by one of two adverbs (moderately, very much). A typical relationship description was:

Ron likes Jan moderately. Jan dislikes Ron very much.

All possible combinations of likes/dislikes and moderately/very much were used for both stimulus persons, thus creating 16 different relational descriptions. The presentation order of the 16 relationships was randomized within each booklet.

Four different forms of the booklet were employed. These forms varied the identity of the stimulus persons. In one form, both were male (Ron and Jim); in one, both were female (Sue and Jan); and in the remaining two, one was male and one was female (Ron and Jan, Sue and Jim). Five male subjects and five female subjects received each booklet. Subjects were assigned randomly to the four conditions.

For each of the relationship descriptions, subjects were asked to respond to 32 statements concerning possible relations between the two stimulus persons by indicating subjective probabilities. Of the 32 statements, 16 were filler statements which were not analyzed. The remaining 16 were statements which implied either dominance or submission. Eight of the statements implied the dominance of the first person over the second (or submission of the second to the first), while eight implied the reverse. The order of all 32 statements was randomly determined but was constant for all subjects.

Subjects responded by assigning each statement a number between 0 and 100 . Subjects were instructed that a rating of 100 indicated absolute certainty that a statement was true and a rating of 0 indicated absolute certainty that a statement was false. A rating of 50 indicated complete uncertainty. Thus, the ratings corresponded to subjective probability judgments. Dominance scores were computed by averaging over the eight sentences that expressed first-person dominance, and then subtracting average second-person dominance from average firstperson dominance.

\section{RESULTS}

The dependent variable, "first-person" dominance, was subjected to a five-factor ANOVA. The factors were sex of subject, sex of first stimulus person, sex of second stimulus person, degree of first-person liking, and degree of second-person liking. If the extent of inferred dominance is a consistent function of asymmetry of liking, then there should be significant main effects for both first-person and second-person liking and no significant interaction of those two variables. These were precisely the effects obtained. The $F$ values and degrees of freedom were $45.21(\mathrm{df}=3 / 96)$ for first-person liking, 34.79 $(\mathrm{df}=3 / 96)$ for second-person liking, and $1.58(\mathrm{df}=$
9/388) for the interaction. None of the other effects were significant.

Substantively, the results indicated that the better liked person was systematically judged to be the more influential. A secondary analysis indicated that not only did the amount of dominance attributed to the better liked person increase as a function of degree of asymmetry, but also that this effect was highly linear. Each of the four simple effects for both first- and secondperson liking was partitioned into linear, quadratic, and residual (higher order) effects, and in each case only the linear component was significant $(p<.01)$. The mean percent of variance accounted for by linearity was 92.79 .

\section{DISCUSSION}

The results of the present study indicate, over a range of conditions, that situations involving asymmetric affective relations were interpreted in terms of dominance or control relations. Regardless of sex of subject or the composition, by sex, of the stimulus dyad, the better liked person was seen as dominating the interaction.

It is quite easy to provide an interpretation of the data in terms of the theoretical position which led to the study. The person maintains a set of expectations concerning affective relations, among which is the expectation of symmetry. Information which is counter to such an expectation provides a stimulus to further explanation. At the same time, the presence of asymmetry is congruent with a relation involving interpersonal dominance. This asymmetry thus provides a cue to an alternative explanation, for the person, which is manifested in terms of an interpretation in terms of dominance. Thus, the person interprets asymmetrical liking as differential dominance.

Such an explanation of the data is by no means the only possible conclusion to be drawn. The fact that it is invariably the better liked person who is seen as the dominant person suggests a more substantively based explanation. Waller and Hill (1951) have argued that the person least interested in the continuation of a given relationship will be the more influential party in that relationship. The present data are congruent with the position that this principle of least interest is axiomatic to naive or implicit theories of interpersonal relationships, so long as one assumes that the person who likes more is thereby more interested in the relationship.

The "puzzle-solving" nature of the task gives rise to a partial explanation in terms of demand characteristics. Such an argument clearly cannot be refuted based on the present experiment. However, this demand characteristics argument does not provide a sufficient interpretation of why the puzzle was solved in the particular way that it was. At most, it suggests some inherent limitations of the generality of the findings.

\section{REFERENCES}

BLuMBERG, H. J. On being liked more than you like. Journal of Personality and Social Psychology, 1969, 11, 121-128.

BuRNSTEIN, E. Sources of cognitive bias in the representation of simple social structures: Balance, minimal change, positivity, reciprocity, and the respondent's own attitude. Journal of Personality and Social Psychology, 1967, 1, 36-48.

Cartwright, D., \& Harary, F. Structural balance: A generalization of Heider's theory. Psychological Review, 1956, 63, 277-293. 
DeSoto, C. B. Learning a social structure. Journal of Abnormal and Social Psychology, 1960, 60, 417-421.

DeSoto, C. B., \& Kuethe, J. L. Subjective probabilities of interpersonal relationships. Journal of Abnormal and Social Psychology, 1959, 59, 290-294.

HEIDER, F. The psychology of interpersonal relations. New York: Wiley, 1958.

Press, A. N., Crockett, W. H., \& Rosenkrantz, D. S. Cognitive complexity and the learning of balanced and unbalanced social structures. Journal of Personality, 1969, 37, 541-553.

Rosenberg, S., \& SedlaK, A. Structural representations of implicit personality theory. In L. Beckowitz (Ed.), Advances in experimental social psychology (Vol. 6). New York: Academic Press, 1972.
WALKER, C. J. The employment of vertical and horizontal social structure in the learning of a social structure. Journal of Personality and Social Psychology, 1976, 33, 132-141.

Waller, W., \& Hill, R. The family. New York: Dryden Press, 1951.

Zajonc, R. B., \& Burnstein, E. Structural balance, reciprocity, and positivity as sources of cognitive bias. Journal of Personality, $1965,33,570-583$.

(Received for publication February 4, 1977.) 